\section{Fat chance for cancer cachexia}

\section{By Lauren Martz, Staff Writer}

With no drugs on the market and a pair of recent Phase III failures in cancer-associated cachexia, companies could be better off turning to the cause of the wasting disease rather than targeting its symptoms. Two independent academic teams have found evidence that browning of white fat is responsible for cachexia in patients with cancer and propose different strategies to block the process.

Last year, GTx Inc.'s enobosarm missed a primary endpoint of improving physical function in a Phase III trial in non-small cell lung cancer (NSCLC), although it met the co-primary endpoint of increasing lean mass. The compound-a selective androgen receptor modulatoris still in testing for cancer. In 2010, Ark Therapeutics Group plc discontinued development of its angiotensin-converting enzyme (ACE) inhibitor, Vitor, after the compound failed in a Phase III trial for cancerassociated cachexia. Both compounds address symptoms by reversing rather than preventing changes that cause the damage.

The new studies, from the Spanish National Cancer Research Centre (CNIO) and Dana-Farber Cancer Institute, provide the first mechanistic links between brown fat activation and cancer cachexia-an association that has been known for over 30 years-and suggest 2 ways to stop the decline.

Jan Nedergaard, a professor of molecular biosciences at Stockholm University, told $S c i B X$ that many studies have suggested that brown fat activation could play a role in cancer cachexia. But, she said, "over the years, a recurrent issue has been the missing molecular link between cancer and the brown adipose tissue."

The CNIO-led group showed that brown fat activation is a consequence of cancer and that anti-inflammatory compounds can block the browning process and decrease cachexia severity. ${ }^{1}$ The DanaFarber group also found that cancer causes browning of white adipose tissue (WAT), but they went a step further to identify a specific tumorsecreted factor, parathyroid hormone-like hormone (PTHLH; PTHRP), that stimulates the conversion and could also serve as a biomarker. ${ }^{2}$ The team is pursuing an antibody against PTHRP for clinical use.

Cachexia is a wasting syndrome associated with several chronic diseases in addition to cancer. It involves decreased food intake as well as an imbalance in energy expenditure that cannot be corrected by supplementing nutrients. But in cancer, the condition also has severe consequences for treatment of the disease.

"Cachexia is not only the cause of a large percentage of cancerassociated deaths, it is also a big reason that chemotherapy has to be stopped and contributes to a poor outcome," said Michele Petruzzelli, a postdoctoral fellow at $\mathrm{CNIO}$ and lead author on the paper.

Although there are no marketed drugs, at least 12 companies have compounds in development for cancer-associated cachexia (see Table 1, "Cancer-associated cachexia products in clinical development").

According to John Beadle, CEO of PsiOxus Therapeutics Ltd., the cachexia therapeutics in development fail to address the cause of the disease. "I believe that the treatments trialed to date have attempted to impact the symptoms of cachexia. Selective androgen receptor modulators, for example, attempt to reverse the loss of muscle, whilst ghrelin analogs attempt to reverse appetite loss. These are certainly signs and symptoms of cachexia, but they are not causative factors," he said. He added that therapeutics targeting the root cause of the disease could lead to improvements not only in muscle mass but also in fat mass and muscle strength.

The two new papers could put the conversion of WAT to brown fat front and center in the search for new therapies.

Unlike WAT, which stores energy as intracellular lipid droplets, brown and beige adipocytes are highly metabolically active and promote energy expenditure by breaking down lipids. White adipocytes can be converted to beige adipocytes by exposure to cold or by activation of $\beta$-adrenergic receptors and some immune-mediated pathways. ${ }^{3,4}$ Although brown adipocytes are not abundant in adults, beige adipocytes are interspersed throughout white tissue.

Both teams set out to explore the molecular basis of brown fat activation in cachexia that had been reported in rodent models of the disease and in some patients. The CNIO study was led by Erwin Wagner, head of CNIO's Genes, Development and Disease team. The DanaFarber team was led by Bruce Spiegelman, a professor of cell biology and medicine at Dana-Farber and Harvard Medical School. Spiegelman is also cofounder of Ember Therapeutics Inc., which works on brown fat activation for metabolic diseases.

\section{The uncoupling link to PTHRP}

Both the CNIO and Dana-Farber studies used mouse models of cancerassociated cachexia to explore the disease mechanism. Whereas the Dana-Farber team used only the Lewis lung carcinoma (LLC) model, the CNIO team used a range of models including genetic, syngenic, chemical-induced and xenograft models of various cancer types. In all models, mice showed the loss of fat and skeletal muscle that is characteristic of cancer-associated cachexia.

Wagner's team showed that WAT browning began before skeletal muscle wasting, which gave the first clue that browning could be a cause of the wasting disease.

Both studies found that uncoupling protein 1 mitochondrial proton carrier (UCP1) was a key player in the process. UCP1 directs mitochondrial respiration toward thermogenesis and away from ATP synthesis and serves as a marker for brown fat activation. Wagner and colleagues demonstrated that UCP1 expression was higher in WAT in the mouse models than in WAT from healthy controls. In addition, the group found UCP 1 expression in 7 of 8 samples of human adipose tissue from patients with colon cancer who have cachexia. No expression was 
Table 1. Cancer-associated cachexia products in clinical development. Although two compounds failed in Phase III trials for cancer cachexia, the landscape is still full of companies working to develop therapeutics for the cancer-associated form of the disease. At least

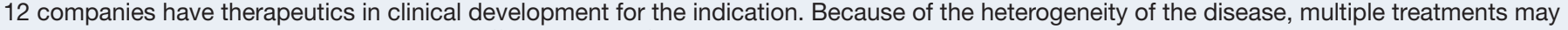
be needed to address cachexia caused by different types of cancer.

Source: BCIQ: BioCentury Online Intelligence

\begin{tabular}{|c|c|c|c|}
\hline Company & Product & Description & $\begin{array}{l}\text { Phase of } \\
\text { development }\end{array}$ \\
\hline $\begin{array}{l}\text { Helsinn Healthcare S.A.; Chugai Pharmaceutical Co. Ltd. } \\
\text { (Tokyo:4519); Especificos Stendhal S.A. de C.V.; Gruppo } \\
\text { Angelini; Ono Pharmaceutical Co. Ltd. (Tokyo:4528); } \\
\text { Specialised Therapeutics Australia Pty. Ltd. }\end{array}$ & Anamorelin & Small molecule ghrelin mimetic & Phase III \\
\hline XBiotech Inc. & CA-18C3 & Human $m A b$ against IL- $1 \alpha$ & Phase III \\
\hline Acacia Pharma Ltd. & APD209 & $\begin{array}{l}\text { Oral fixed-dose combination of progestin and the } \\
\text { selective adrenergic receptor } \beta_{2} \text { (ADRB2) agonist } \\
\text { formoterol }\end{array}$ & Phase II \\
\hline Aeterna Zentaris Inc. (TSX:AEZ; NASDAQ:AEZS) & AEZS-130 & $\begin{array}{l}\text { Oral ghrelin mimetic that acts as a growth hormone } \\
\text { secretagogue }\end{array}$ & Phase II \\
\hline Akela Pharma Inc. & GHRH analog & $\begin{array}{l}\text { Peptide analog of growth hormone-releasing hormone } \\
\text { (GHRH) }\end{array}$ & Phase II \\
\hline Alder Biopharmaceuticals Inc. (NASDAQ:ALDR) & Clazakizumab & Humanized $\mathrm{mAb}$ against IL-6 & Phase II \\
\hline Novartis AG (NYSE:NVS; SIX:NOVN) & Bimagrumab & Human $\mathrm{mAb}$ against activin receptor type $2 \mathrm{~B}$ (ACVR2B) & Phase II \\
\hline Ohr Pharmaceutical Inc. (NASDAQ:OHRP) & OHR/AVR118 & Broad-spectrum peptide nucleic acid immunomodulator & Phase II \\
\hline PsiOxus Therapeutics Ltd. & MT-102 & $\begin{array}{l}\text { Dual-action anabolic and catabolic transforming agent } \\
\text { with ADRB antagonist activity }\end{array}$ & Phase II \\
\hline \multirow{2}{*}{ Rose Pharma A/S } & GTP-200 & Ghrelin-based compound & \multirow{2}{*}{ Phase II } \\
\hline & GTP-300 & Ghrelin peptide & \\
\hline Vicus Therapeutics LLC & VT-122 & $\begin{array}{l}\text { Fixed-dose combination of propranolol, a nonselective } \\
\text { ADRB blocker, and the cyclooxygenase-2 (COX-2) } \\
\text { inhibitor etodolac }\end{array}$ & Phase II \\
\hline Ligand Pharmaceuticals Inc. (NASDAQ:LGND) & YK5211 & Nonsteroidal selective androgen receptor modulator & Phase I \\
\hline
\end{tabular}

found in adipose tissue samples from 20 patients with cancer who do not have cachexia.

In Spiegelman's study, cultured media from LLC cells induced UCP1 expression in white adipocytes, which suggested that cancer cells secrete factors that promote adipose tissue browning. With this in mind, the researchers looked for secreted proteins that were specifically upregulated in highly thermogenic LLC clones and identified PTHRP as a potential stimulator of WAT browning.

Next, the Spiegelman team tested a neutralizing antibody against PTHRP in the LLC model of cachexia. The antibody prevented weight loss and blocked adipose tissue and skeletal muscle wasting, and it decreased energy expenditure compared with control antibody.

Finally, the Dana-Farber group showed that PTHRP could be detected in the serum of 17 out of 47 patients with NSCLC or colon cancer. Those patients had lower lean body mass and resting energy expenditure than patients in whom PTHRP was undetectable.

The Dana-Farber study was published in Nature.

Spiegelman said that his team plans to develop a humanized antibody or other reagent that could neutralize human PTHRP. "I can't see any obvious disadvantage, as humans should not really have circulating levels of PTHRP. That said, you never know until it is tested in humans," he said.

His team is also looking into whether PTHRP is involved in cachexia caused by other types of cancers and other diseases including kidney and heart disease.

\section{An inflammatory cause}

Because inflammation occurs with cancer, Wagner's team used a syngenic mouse model of skin cancer to study whether inflammation was linked to cachexia and found that levels of IL- 6 and other inflammatory markers were higher than those in healthy controls.

IL- $6^{+}$colon cancer cells implanted into mice caused tumor formation and cachexia, whereas IL- $6^{-}$cells caused tumors but no cachexia. Because IL-6 is involved in proinflammatory responses, the results suggested that inflammation could contribute to the induction of cachexia.

To test that hypothesis, Wagner's group looked at the effect of an NSAID in the skin tumor model. Sulindac, a cyclooxygenase-2 (COX2) inhibitor, decreased browning of subcutaneous WAT and reduced cachexia severity compared with vehicle.

Petruzzelli said that the team is now looking for new compounds that could inhibit the browning process based on this research.

"We found a strong anticachexic effect from NSAIDs, but chronic treatment is not indicated because of potentially serious side effects such as gastrointestinal bleeding," said Petruzzelli. "It is known, for example, that NSAIDs may help prevent intestinal cancer, but they are not used for the indication because the side effects outweigh the benefits."

He added, "We guess that patients would need very long treatment likely before cachexia manifests. Patients may take between 6 months and 5 years before developing cachexia, and at this moment there is no way to predict if and when it will develop. We need a very safe therapeutic or a biomarker to predict which patients will develop cachexia and when it will present to justify treatment with more risks."

His team is also trying to identify a disease-specific biomarker to guide treatment.

Data were published in Cell Metabolism. 


\section{ANALYSIS}

\section{Disease heterogeneity}

Although researchers agree that both teams have presented promising new approaches to pursue in the cancer cachexia space, the key to

\section{"Cachexia is a highly}

heterogeneous disease. While we do know that in terms of the mechanism there is some degree of commonality between cachexia caused by different diseases, we need to separate cancer-associated cachexia, and maybe even cachexia caused by individual types of cancer, from other causes of the disease for clinical trial and drug development purposes." -Michele Petruzzelli, Spanish National Cancer Research Centre

clinical success with any strategy may be careful patient stratification.

"Cachexia is a highly heterogeneous disease. While we do know that in terms of the mechanism there is some degree of commonality between cachexia caused by different diseases, we need to separate cancerassociated cachexia, and maybe even cachexia caused by individual types of cancer, from other causes of the disease for clinical trial and drug development purposes," said Petruzzelli.

He added that the work in the Nature paper is particularly interesting because screening patients for PTHRP expression could help select patients likely to respond to a therapeutic that targets the biomarker.

The heterogeneity of cachexia in different patients with cancer could be another reason that therapeutic efficacy has been difficult to prove in Phase III testing. Although it is not yet clear whether targeting a general mechanism such as inflammation could be effective in patients with cachexia, some researchers think it might be necessary to design separate therapeutics for patients with cachexia who have different kinds of cancer or different factors contributing to brown fat activation.
The Dana-Farber team has already identified PTHRP as a therapeutic target that could help a subset of patients with cancer cachexia and serve as a way to identify that subset.

Spiegelman told SciBX that his team plans to apply the same strategy used to identify PTHRP in the lung cancer model to other cancer cachexia models to find factors involved in the browning process of other cancers.

Spiegelman's company Ember has no plans to pursue therapeutics for cachexia based on this work. Dana-Farber has filed a patent application covering the work, and the IP is available for licensing. The work from the CNIO group is unpatented, but the team is interested in partnering to develop anticachexia therapeutics.

Martz, L. SciBX 7(32); doi:10.1038/scibx.2014.941

Published online Aug. 21, 2014

\section{REFERENCES}

1. Petruzzelli, M. et al. Cell Metab.; published online July 17, 2014; doi:10.1016/j.cmet.2014.06.011

Contact: Erwin F. Wagner, Spanish National Cancer Research Centre (CNIO), Madrid, Spain e-mail: ewagner@cnio.es

2. Kir, S. et al. Nature; published online July 13, 2014; doi:10.1038/nature13528

Contact: Bruce M. Spiegelman, Harvard Medical School, Boston, Mass.

e-mail: bruce_spiegelman@dfci.harvard.edu

3. Cao, L. et al. Cell Metab. 14, 324-338 (2011)

4. Lou, K.-J. SciBX 7(26); doi:10.1038/scibx.2014.753

\section{COMPANIES AND INSTITUTIONS MENTIONED}

Ark Therapeutics Group plc (LSE:AKT), London, U.K.

Dana-Farber Cancer Institute, Boston, Mass.

Ember Therapeutics Inc., Boston, Mass.

GTx Inc. (NASDAQ:GTXI), Memphis, Tenn.

Harvard Medical School, Boston, Mass.

PsiOxus Therapeutics Ltd., Abingdon, U.K.

Spanish National Cancer Research Centre, Madrid, Spain

Stockholm University, Stockholm, Sweden 